\title{
Dans la tête de Papa
}

\author{
Concept et texte : Livia Edery \\ Illustrations : Pastan a.k.a. Tristan Ney \\ Publié par ARTC sud et l'Assistance publique des Hôpitaux de Marseille
}

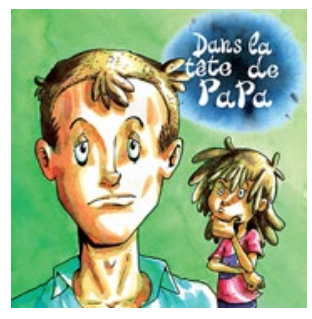

Nous avons à de nombreuses reprises mis en avant toutes les publications « aidantes ». Les plaquettes, les contes, les $\mathrm{BD}$, tout ce qui permet aux enfants et à leurs accompagnants de parler du cancer, des traitements, de la vie avec cancer et parfois aussi de la mort après cancer.

Livia Edery, psychologue dans le service de neurooncologie du Pr Olivier Chinot, nous propose un livret pour les enfants intitulé «Dans la tête de Papa».

Que se passe-t-il dans la tête de papa depuis quelque temps? Trop fatigué pour jouer avec moi, bizarre, patraque... Hier maman et lui sont rentrés de la consultation chez le médecin sans un mot, hébétés. Mal à la tête, tout est dans la tête, casse-tête...

L'enfant au premier plan sur les illustrations de Pastan est perplexe. Nous voilà en effet en caméra subjective : comment comprendre les adultes qui ne jouent plus leur rôle et qui sont eux-mêmes dépassés par les événements ?

Ils parlent d'IRM? Qu'à cela ne tienne, en voici l'illustration: papa dans la machine avec ses lunettes de soleil sous les flashs. Et voici des images sombres avec le crâne de papa qui forment autant de drapeaux sombres de pirate. Puis le médecin qui n'est plus qu'une bouche qui commente les images, laissant les parents cois devant l'indicible: "le médecin appelle ça une tumeur cérébrale». Suit une explication très imagée de la « boule » en question. Tous les enfants comprendront, même si le mot cancer n'est jamais prononcé. Les traitements suivent et leurs effets ne se ressemblent pas. Très parlantes ces images, même un peu violentes, mais cette affaire de tumeur cérébrale ne fait pas dans la dentelle, et les enfants qui se plaignent d'être souvent tenus à part vont avoir de quoi tenir la dragée haute à tous les petits films d'horreur dont ils raffolent.

On comprendra que ce livret est plutôt destiné aux enfants à partir de huit ans, mais bien sûr les plus petits y auront accès avec leurs frères et sœurs, aussi il est important que les psychologues et les soignants ne remettent pas ce livret comme ils font des livrets d'accueil. La remise de ce livret nécessite de le présenter aux parents et de voir s'ils pourront en parler avec leur enfant. La meilleure solution étant de le présenter aux parents et aux enfants en même temps et d'écouter leurs commentaires. La conclusion du livret est importante, car «même si, à cause de la maladie, on ne joue pas aux mêmes jeux qu'avant, mon papa m'aime comme avant ». La maladie grave est souvent dévastatrice dans les familles : absence de parole, représentations négatives, jugements, angoisses. Les enfants absorbent tout et souvent se taisent... Dans ce petit recueil, les difficultés sont abordées telles que les enfants les ressentent : souvent discontinues, éphémères ou au contraire brutales dans de longues périodes d'attente.

Pas facile de parler de ce qu'il y a dans la tête de papa, parce que justement l'enfant n'est pas dans la tête des grands... 